\title{
Dieter Herberg
}

\section{Neologismen in der deutschen Gegenwartssprache Probleme ihrer Erfassung und Beschreibung}

\section{$1 \quad$ Einstieg}

Ungeachtet des starken Interesses der Öffentlichkeit an aktuellen und schnellen Informationen über Neues im Wortschatz, das sich z. B. in einer Fülle von Miszellen, Glossen, Beispielsammlungen $u$. Ä. in Zeitungen und Zeitschriften spiegelt, spielt der Neologismus in der germanistischen Linguistik - unter lexikologischem wie unter lexikografischem Gesichtspunkt - eine vergleichsweise geringe Rolle. Die erheblichen und des Öfteren benannten Defizite, die in der germanistischen Neologismenforschung und Neologismenlexikografie bis heute bestehen (vgl. z. B. Heller u. a. 1988; Herberg 1988; Wiegand 1990; Kinne 1996; Herberg/Kinne 1998), gaben den Anstoß dazu, die Neologismenforschung Mitte der 90er-Jahre im Forschungsplan des Instituts für Deutsche Sprache (IDS) in Mannheim als langfristige Aufgabe zu etablieren. In diesem Rahmen wurde 1997 mit den Arbeiten am ersten größeren, den Prinzipien der wissenschaftlichen Lexikografie verpflichteten genuinen Neologismenwörterbuch - nämlich dem für die Allgemeinsprache der 90er-Jahre des 20. Jahrhunderts - begonnen. Zunächst als Printwörterbuch geplant, wurde im Zusammenhang mit der Konzipierung des lexikalisch-lexikologischen, korpusbasierten Informationssystems „Wissen über Wörter“ (WiW) im IDS das Neologismenprojekt als Pilotprojekt für WiW umkonzipiert, sodass nun das primäre Ziel darin besteht, bis 2005 das lexikografische Informationsangebot zu den Neologismen als elektronische Datenbank im Rahmen von WiW der allgemeinen Nutzung per Internet zur Verfügung zu stellen. Im Folgenden beziehen wir uns auf dieses Neologismenprojekt (vgl. dazu auch Herberg 2001; Tellenbach 2002).

\section{Zum Begriff des Neologismus}

Bis heute zeigen Definitionen von „Neologismus" in der Fachliteratur eine auffällige Vagheit und Uneinheitlichkeit. Für die Definition des Neologismus, die wir unserem Projekt zugrunde gelegt haben, bildet das bilaterale Zeichen aus Ausdrucks- und Inhaltsseite, also aus Form und Bedeutung, den Ausgangspunkt. Von den seit langem etablierten Wortschatzeinheiten unterscheidet sich der Neologismus dadurch, dass entweder die Form und die Bedeutung oder nur die Bedeutung der betreffenden Einheit von der Mehrheit der Angehörigen einer bestimmten Kommunikationsgemeinschaft eine Zeit lang als neu empfunden wird. Wir unterscheiden also zwei Typen von Neologismen: Neulexeme und Neubedeutungen. Der naturgemäß gegebene Bezug zu der jeweiligen Zeit des Aufkommens macht den Neologismus-Begriff zu einem relativen und historisch gebundenen. Die Bezugnahme auf den (mehr oder weniger exakt zu bestimmenden) Zeitpunkt des Aufkommens sowie auf die sich anschließende Ausbreitungs- und Durchsetzungsphase ist für die Definition des Neologismus konstitutiv. Unter Berücksichtigung der genannten Gesichtspunkte kann der Neologismus in folgender Weise definiert werden: Ein Neologismus ist eine lexikalische Einheit bzw. eine Bedeutung, die in einem bestimmten Abschnitt der Sprachentwicklung in einer Kommunikationsgemeinschaft aufkommt, sich ausbreitet, als sprachliche Norm allgemein akzeptiert und in diesem Entwicklungsabschnitt von der Mehrheit der Sprachbenutzer über eine gewisse Zeit hin als neu empfunden wird.

Im gegebenen Fall entspricht der so genannte „Erfassungszeitraum“ den 90er-Jahren 
des 20. Jahrhunderts. Abgesehen vom Aktualitätsargument wurden die $90 \mathrm{er}-J a h r e$ aus verschiedenen weiteren Gründen gewählt. Ein Zeitraum von zehn Jahren erschien für den ersten Angang deutscher Neologismenlexikografie sinnvoll, weil er weder zu knapp noch zu umfangreich bemessen und damit überschaubar ist. Den zeitlichen Ausgangs- und Endpunkt des Jahrzehnts bilden markante Daten: der 3. Oktober 1990 mit der Herstellung der Einheit Deutschlands; der Abschluss des letzten Jahrzehnts des 20. Jahrhunderts und damit das Ende eines Jahrtausends.

\section{Das lexikografische Neologismenprojekt}

Ziel des Projekts ist es, den im In- und Ausland großen Informationsbedarf in Bezug auf neue Wörter und Bedeutungen des Deutschen zu befriedigen. Es stellt die Neologismen dar, die sich im allgemeinsprachlichen Teil des Wortschatzes der deutschen Standardsprache in den 90er-Jahren etabliert haben. Die Wortartikel enthalten neben der Bedeutungsangabe, Kollokationen und einer Belegauswahl u. a. Angaben zum jeweiligen Typ des Neologismus, zur Herkunft, zur Gebrauchsspezifik und zur Wortbildung des betreffenden Stichwortes.

Einige Ausführungen zur Materialbasis, zur Stichwortauswahl und zur Präsentationsform sollen das Projekt genauer erläutern.

\subsection{Die Materialbasis}

Was die Erhebung des Sprachmaterials betrifft, so gilt grob die folgende Schwerpunktverteilung: Die gezielte Suche nach Neologismen erfolgt durch die Projektmitarbeiter in Primärquellen aller Art wie auch in Sekundärquellen; die Menge der Belegungen für die gefundenen Neologismen und für die ausdrucksseitigen „Familienangehörigen“ liefert uns das elektronische Textkorpus. Das elektronische Korpus, das als sog. virtuelles Korpus „neo“ als Teil der IDS-Korpora geschriebener Sprache zur Verfügung steht, umfasst Zeitungstexte der 90er-Jahre (z. B. Berliner Zeitung, Frankfurter Allgemeine, Mannheimer Morgen, Der Spiegel, die tageszeitung, Die Zeit, Frankfurter Rundschau, Süddeutsche Zeitung). Hinzu kommt die projekteigene Wortkartei, die ca. 10.000 subjektiv ausgewählte, durch Exzerption gewonnene Belege aus verschiedensten Texten der 90er-Jahre sowie Hörbelege aus Texten von Fernseh- und Rundfunksendungen dieses Zeitraumes enthält.

\subsection{Die Stichwortauswahl}

Der lange und nicht einfache Weg zu einer begründeten Stichwortauswahl für das Projekt ist an anderer Stelle ausführlich dargestellt worden (Herberg 2001; 2002). Die für die Bearbeitung ausgewählten lexikalischen Einheiten müssen drei Kriterien genügen. Sie müssen

1. dem deutschen Sprachgebrauch entsprechen (d. h. in Bezug auf den hohen Anteil von Einheiten angloamerikanischer Herkunft deren Übernahme in den deutschen Sprachgebrauch als Angloamerikanismen mit der entsprechenden Assimilation von Schreibung und Flexion);

2. neu für die 90er-Jahre sein;

3. der Allgemeinsprache angehören (d.h., dass rein fach- und gruppensprachliche Lexik ebenso wie Regionales ausgeklammert bleiben).

Als Selektionsresultat bleiben etwa 800 Neologismen, die den drei Auswahlkriterien entsprechen. Wie setzt sich diese Stichwortauswahl unter sprachinternem und unter sprachexternem Aspekt zusammen?

Unter sprachinternem Aspekt ist festzustellen, dass ein bemerkenswert hoher Anteil von ca. 40 Prozent auf Lexeme entfällt, die aus dem Englischen bzw. Amerikanischen entlehnt sind und die wir hier als „Angloneologismen" bezeichnen wollen. Bei weiteren 20 Prozent handelt es sich um Zusammensetzungen, deren eine Konstituente ein solcher Angloneologismus ist (z. B. Event in Eventkultur). Was die Wortartzugehörigkeit betrifft, ergibt sich, dass es sich beim Löwenanteil (ca. 85 Prozent) um Substantive handelt; 10 Prozent entfallen auf Verben und nur 3 Prozent auf Adjektive. Der Rest vertritt andere Wortarten (z. B. ups als Interjektion).

Unter sprachexternem Aspekt haben wir die Stichwörter nach Fach-/Sachgebieten geordnet. Die am stärksten besetzten Gebiete sind:

- Computer (z. B. Datenautobahn, Doppelklick, E-Mail),

- Medien (z. B. Bezahlfernsehen, Dailysoap, Multiplexkino),

- Soziales/Gesellschaft (z. B. Bürgergeld, Erlebnisgesellschaft, Mobbing), 
- Sport (z. B. Bungeespringen, Inlineskating, walken),

- Wirtschaft (z. B. E-Commerce, Globalplayer, Outsourcing).

Im Mittelfeld rangieren:

- Bank-/Finanzwesen (z. B. Direktbanking, Electronic Cash, Euro),

- Freizeit/Unterhaltung (z. B. abhängen, Infotainment, Konsolenspiel),

- Arbeitswelt/Bildung (z. B. Assessmentcenter, Mobilzeit, Telelearning),

- Mode (z. B. Basecap, Outdoorjacke, piercen),

- Verkehr/Auto (z. B. Elchtest, Jobticket, Wegfahrsperre),

- Telefon/Telekommunikation (z. B. Call-byCall, Handy, Mobilnetz).

Eher schwach vertreten sind Neologismen aus folgenden Bereichen:

- Gesundheit (z. B. Knopflochchirurgie, Potenzpille, Wellness),

- Musik (z. B. Kuschelrock, Techno, unplugged),

- Nahrungs-/Genussmittel (z. B. Energydrink, Gentomate, Partydroge).

Weder die Zahl oder die Bezeichnung der Gebiete noch die Relationen zwischen den Gruppen sind objektivierbar. Es ist aber nicht zu bezweifeln, dass in Abhängigkeit vom jeweiligen Erfassungszeitraum und von der gesellschaftlichen Gesamtsituation der Einfluss bestimmter Fach- und Sachgebiete auf die Allgemeinsprache dominiert.

Mit Sicherheit ist es ein Spiegelbild von Entwicklungen der 90er-Jahre, dass nach unseren Erhebungen Lexik aus der Sphäre des Computers und der Medien, aus dem Sozialund dem Wirtschaftsbereich und aus dem Sport den Allgemeinwortschatz besonders stark erweitert.

\subsection{Die Präsentationsform}

Es kann in diesem Rahmen nicht ausführlich auf die durch das Informationssystem „Wissen über Wörter" gegebene und auch für das Neologismenprojekt verbindliche Präsentationsform eingegangen werden. Nur so viel sei gesagt: Eine der DV-Komponenten, aus denen WiW besteht, ist eine objektrelationale Datenbank. In dieser Datenbank sollen die Ergebnisse aller wortschatzbezogenen Projek- te des IDS sowie mittelfristig auch Ergebnisse externer Wortschatzforschungen abgelegt, gebündelt und in Beziehung zueinander gesetzt werden um so zu neuem linguistischem Wissen vorstoßen zu können (vgl. dazu НаßZumkehr 2000).

In der gegenwärtigen Entwicklungs- und Erprobungsphase zu WiW ist die Neologismenprojektgruppe - entsprechend der ihr zugedachten Pilotfunktion - dabei, mittels eines XML-Editors (XMetaL) systematisch die Wortartikel zu den rund 800 ausgewählten Neologismen zu erarbeiten. Die Anordnung und Strukturierung lexikalischer Informationen weicht in diesem Präsentationsmedium zum Teil stark von dem ab, was man von gedruckten Wörterbüchern her kennt. Die Systematisierung der Daten und ihre Eintragung in die Eingabemaske des Editors wird durch fünf vorgegebene Informationsdimensionen gesteuert:

- Schreibung und Aussprache,

- Bedeutung und Verwendung,

- Grammatik,

- Geschichtliches und Sachliches,

- Kritisches und Normatives.

Alle Daten, also Wörter oder Textteile, die vom Lexikografen eingetragen werden, sind in sog. Tags eingeschlossen, die sie als zu einer bestimmten Angabeart gehörend markieren, wodurch künftig die automatische Suche oder Sortierung nach der betreffenden Angabeart ermöglicht wird.

Die konkrete Darstellung stellt den Lexikografen bei nahezu jedem Neologismus in Bezug auf die eine oder andere, oft auch in Bezug auf mehrere der Informationsdimensionen vor Probleme. Das gilt insbesondere für die Angloneologismen und für Zusammensetzungen mit ihnen, haben sich doch bei ihnen Schreib-, Aussprache-, Gebrauchs- und grammatische Normen häufig noch nicht gefestigt, sodass Varianten auftreten können, die einen unterschiedlichen Grad der Integration in das deutsche Normensystem zeigen.

Im Folgenden soll am Beispiel der unter den Neologismen dominierenden Wortart Substantiv auf einige der diesbezüglichen Normunsicherheiten innerhalb der Informationsdimensionen „Schreibung und Aussprache" und "Grammatik" eingegangen werden. die sich bei Angloneologismen ergeben können. 


\subsection{Schreibvarianten}

Neologismen treten uns in den Korpustexten oft in unterschiedlichen Schreibungen entgegen. Besonders bei Angloneologismen können auffallend viele Schreibvarianten vorkommen, die einen unterschiedlichen Grad der Integration in die deutsche Schreibnorm zeigen, z. B. Carsharing ('das Nutzen eines Autos durch verschiedene Personen auf organisierter, meist kommerzieller Grundlage') mit den Varianten Car-Sharing, CarSharing, Car-sharing, Car Sharing, carsharing, car-sharing. Grundsätzlich werden alle in den Texten belegten Schreibvarianten eines Neologismus verzeichnet.

Der Lemmaansatz erfolgt entsprechend den Regeln der neuen deutschen Rechtschreibung. Im vorliegenden Beispiel - also bei nominalen Zusammensetzungen - ist die Norm die Zusammenschreibung, die mithin als sog. Lemmazeichengestaltangabe fungiert: Carsharing. Innerhalb der Informationsdimension "Schreibung und Aussprache" werden die Varianten in folgender Weise behandelt:

Am Anfang stehen - sofern vorhanden Normvarianten. Bei nominalen Zusammensetzungen ist in der Regel die Bindestrichschreibung mit der zweiten Konstituente in Großschreibung Normvariante: Car-Sharing. Sie wird übrigens auch dann angegeben, wenn sie weder im Korpus belegt noch in einem Kanon von festgelegten Vergleichswörterbüchern aus dem Erfassungszeitraum gebucht ist. Häufig gibt es - wie oben gezeigt - neben den Normschreibungen eine Anzahl von Nicht-Normvarianten zu registrieren. Für deren Anordnung kann nach unserer Auffassung nicht das Prinzip der Beleghäufigkeit angewandt werden, weil sich nach unseren Beobachtungen durch Korpuserweiterungen wechselnde Rangfolgen ergeben (können). Wir haben uns daher für ein Anordnungsprinzip entschieden, dem die Struktur der Varianten zugrunde liegt. Generell gilt: Großschreibung der ersten Konstituente ist - entsprechend der generellen Substantivgroßschreibung im Deutschen - das übergeordnete Kriterium und rangiert vor Kleinschreibung. Innerhalb der initialen Groß- bzw. Kleinschreibung werden - gemäß der Normschreibung des Lemmazeichens - zunächst weitere Zusammenschreibungen (CarSharing, carsharing), darauf weitere Bindestrichschreibungen (Car-sharing, car-sharing) und zuletzt die den deutschen Schreibnormen für nominale Zusammensetzungen am fernsten stehenden Getrenntschreibungen (Car Sharing) aufgeführt. Das mag genügen. Es ging lediglich darum, an einem Beispiel zu zeigen, dass sich bei den Angloneologismen bereits in der die Ausdrucksseite betreffenden Informationsdimension "Schreibung und Aussprache" Probleme der lexikografischen Beschreibung ergeben, die sich so zugespitzt beim indigenen Wortschatz nicht stellen.

\subsection{Varianten beim Genus}

Das Problem der Genuszuweisung tritt bei Angloneologismen immer wieder auf (vgl. auch Steffens 2002). Es ist bekannt, dass die Genuszuweisung bestimmten Prinzipien folgt, so z. B. dem der lexikalischen Ähnlichkeit (der Shareholdervalue - der Wert/der Profit), dem des natürlichen Geschlechts ( $\underline{\text { der }} A n$ chorman), dem der morphologischen Analogie (z. B. sind Substantive mit dem Suffix -ness Feminina: die Political Correctness, die Wellness) u. a. (vgl. dazu Schlick 1984; 1984/85).

Oft ist das Ergebnis aber nicht eindeutig, sondern wir sind mit zwei (z. B. Event, Mail), mitunter auch mit allen drei Genera (z. B. Couchpotato) bei ein und demselben Neologismus konfrontiert. Im Unterschied zu den Schreibvarianten werden mehrere Genera nach Vorkommenshäufigkeit geordnet, wobei das erstgenannte, gut belegte Genus unkommentiert bleibt, während beim schwach belegten zweiten (und ggf. dritten) Genus ein Verwendungshinweis gegeben wird. Als Beispiel diene Couchpotato ('jemand, der seine Freizeit vor allem vor dem Fernseher verbringt und dabei häufig Süßigkeiten, Salzgebäck isst'): Das am stärksten belegte Genus ist das Femininum; es folgen Genus Maskulinum und Genus Neutrum mit dem Verwendungshinweis "Genus Maskulinum selten“" bzw. "Genus Neutrum selten".

Die Genuszuweisung wird nur beim Genus Femininum kommentiert, da sie sich durch das Genus der deutschen Entsprechung erklären lässt, also dem Prinzip der lexikalischen Ähnlichkeit folgt: „Das Genus Femininum ist wahrscheinlich analog zum Genus Femininum der deutschen Entsprechung von engl. potato, Kartoffel, gebildet.“ 


\subsection{Varianten beim Genitiv Singular}

Varianten beim Genitiv Singular treten auf bei den zahlreichen Angloneologismen mit Genus Neutrum, bei denen es sich aus Sicht der englischen Wortbildung um Verbalsubstantive mit dem Suffix -ing handelt. Der Gebrauch schwankt zwischen der endungslosen Form (des Carsharing), die der englischen Flexion entspricht, und der Form mit der Endung -s (des Carsharings), die der deutschen Flexion entspricht. Während nun in den allgemeinsprachlichen bzw. Fremdwörterbüchern unseres Kanons von Vergleichswörterbüchern der 90er-Jahre, in denen entsprechende Neologismen gebucht sind, überwiegend der Genitiv mit der Endung $-s$ kodifiziert ist, zeigt die Beleglage im IDS-Textkorpus, dass damit nicht der tatsächliche Sprachgebrauch wiedergegeben wird. Der Befund für die im Genitiv Singular belegten Lexeme auf -ing ist: Nur 16 Prozent kommen ausschließlich mit der Endung -s vor (z. B. bei Bungeejumping), 37 Prozent nur endungslos (z. B. bei Canyoning, Downsizing, Outsourcing), 47 Prozent aber sind in beiden Formen belegt (z. B. bei Banking, Piercing) (vgl. zu diesen Angaben Tellenbach 2002: 112f.). Dieser Befund, aber auch die Tatsache, dass in den Korpustexten Genitiv-Singular-Vorkommen rar sind, wodurch sich die Bedeutung des ausschließlichen Auftretens nur einer der beiden Formen relativiert, legen es nahe, bei diesen Angloneologismen in der Regel sowohl den Genitiv mit der Endung $-s$ als auch den endungslosen Genitiv - in dieser Reihenfolge - anzugeben.

\subsection{Varianten beim Nominativ Plural}

Das zum Nebeneinander von Genitiv-Singular-Formen Gesagte gilt analog für das Nebeneinander von Nominativ-Plural-Formen. In Fällen wie Callcenter, Flyer, Netizen haben wir es sowohl mit der endungslosen Form (die Flyer) als auch mit der auf -s endenden Form (die Flyers) zu tun. In der Regel ist der Nominativ Plural analog zu den meisten deutschen Maskulina mit der Endung -er endungslos und nur selten wird die der englischen Pluralbildung gemäße Form mit der Endung $-s$ verwendet.

In einem Fall wie Flyer, für den in den Vergleichswörterbüchern, in denen dieses Lexem gebucht ist, ausschließlich der Nominativ Plural Flyer angegeben ist, wird in einem Kommentar darauf hingewiesen, dass die Form Flyers nur in unserem Korpus belegt ist: „Nominativ Plural Flyers im Korpus belegt, nicht jedoch in den Wörterbüchern gebucht.“

\section{Schluss}

Mit diesen wenigen Einblicken in die Werkstatt eines als Teil der künftigen WiW-Datenbank entstehenden Internet-Wörterbuches sollen die Ausführungen über Neologismen in der deutschen Gegenwartssprache abgeschlossen werden. Es liegt in der Natur der Sache, dass in Bezug auf etwas, das neu ist, besonders großer Informationsbedarf besteht - das gilt für sprachliche Innovationen genauso wie etwa für technische oder gesellschaftliche. Information tut also Not. Dabei geht es nicht nur allgemein darum, etwa mit Wörterbüchern die aktive und passive Sprachkompetenz der Angehörigen einer Kommunikationsgemeinschaft zu erweitern und zu stärken; für zahlreiche Berufs- und andere soziale Gruppen sind möglichst genaue Kenntnisse über Inhalt, Form und Gebrauchsweise neuer Lexik eine dringliche Notwendigkeit, denkt man z. B. an Journalisten, Übersetzer, Dolmetscher, Lexikografen allgemeiner einsprachiger und zweisprachiger Wörterbücher. Fundierte Hilfe will ein Informationsmittel wie das in Arbeit befindliche genauso Lehrern, Schülern und Studenten auf dem Gebiet Deutsch als Fremdsprache bieten (vgl. Herberg 1997). Dieser Nutzerkreis dürfte es besonders begrüßen, dass die elektronische Präsentationsform laufende Ergänzungen um neueste Neologismen möglich macht und dass die IDS-Neologismengruppe darüber hinaus die Publikation kommentierter Wortlisten mit aktuellen Neologismen von jeweils zwei oder drei Jahren als Material für die schnelle Information plant. 


\section{Literatur}

Haß-Zumkehr, Ulrike (2000): Wortschatz ist mehr als „viele Wörter“. Die Aufgaben der Abteilung Lexik des IDS. In: Sprachreport 2, 2-7.

Heller, Klaus u. a. (1988): Theoretische und praktische Probleme der Neologismenlexikographie. Überlegungen und Materialien zu einem Wörterbuch der in der Allgemeinsprache der DDR gebräuchlichen Neologismen. Berlin (Linguistische Studien. A, 184).

Herberg, Dieter (1988): Stand und Aufgaben der Neologismenlexikographie des Deutschen. In: G. Harras (Hg.), Das Wörterbuch - Artikel und Verweisstrukturen. Düsseldorf, 265-283 (Sprache der Gegenwart, 74; IDS-Jahrbuch 1987).

Herberg, Dieter (1997): Neologismen im allgemeinen Wörterbuch oder Neologismenwörterbuch? Zur Lexikographie von Neologismen. In: K.-P. Konerding/A. Lehr (Hg.), Linguistische Theorie und lexikographische Praxis. Tübingen, 61-68 (Lexicographica. Series Maior, 82).

Herberg, Dieter (2001): Neologismen der Neunzigerjahre. In: G. Stickel (Hg.), Neues und Fremdes im deutschen Wortschatz. Aktueller lexikalischer Wandel. Berlin/New York, 89-104 (IDSJahrbuch 2000).

Herberg, Dieter (2002): Der lange Weg zur Stichwortliste. Aspekte der Stichwortselektion für ein allgemeinsprachliches Neologismenwörterbuch. In: U. Haß-Zumkehr u. a. (Hg.), Ansichten der deutschen Sprache. Festschrift für Gerhard Stickel zum 65. Geburtstag. Tübingen, 237-250 (Studien zur deutschen Sprache, 25).
Herberg, Dieter/Kinne, Michael (1998): Neologismen. Heidelberg (Studienbibliographien Sprachwissenschaft, 23).

Kinne, Michael (1996): Neologismus und Neologismenlexikographie im Deutschen. Zur Forschungsgeschichte und zur Terminologie, über Vorbilder und Aufgaben. In: Deutsche Sprache 4, 327-358.

Schlick, Werner (1984): Die Kriterien für die deutsche Genuszuweisung bei substantivischen Anglizismen. In: The German Quarterly 3, 402-431.

Schlick, Werner (1984/85): „Diese verflixte englische Geschlechtslosigkeit!“ Zur deutschen Genuszuweisung bei neueren Lehnsubstantiven aus dem Englischen. In: Muttersprache 3-4, 193-221.

Steffens, Doris (2002, i. Dr.): Neologismen im Deutschen = Angloamerikanismen?

Tellenbach, Elke (2002): Neologismen der neunziger Jahre. Vom Textkorpus zur Datenbank. In: I. Barz u. a. (Hg.), Das Wort in Text und Wörterbuch. Leipzig, 105-118 (Abhandlungen der Sächsischen Akademie der Wissenschaften zu Leipzig. Philologisch-historische Klasse, Bd. 76, H. 4).

Wiegand, Herbert Ernst (1990): Neologismenwörterbücher (= Kapitel 2.4.1 von: Die deutsche Lexikographie der Gegenwart). In: F. J. Hausmann u. a. (Hg.), Wörterbücher - Dictionaries Dictionnaires. Ein internationales Handbuch zur Lexikographie. 2. Teilbd. Berlin/New York, 2185-2187 (HSK 5.2). 